\title{
How lethal is SARS-CoV-2 pneumonia when compared with respiratory syncytial virus and influenza in young children?
}

\section{James Shunxian Wei}

\section{Background and objective \\ SARS-CoV-2 is known to cause milder disease in children when compared with adults, but the extent of this is unclear. The aim of this article is to estimate the case fatality rate (CFR) for SARS-CoV-2 infection and SARS-CoV-2 pneumonia in young children aged $<5$ years, and compare this with estimated CFRs for respiratory syncytial virus (RSV) and influenza.}

\section{Methods}

This article reviews published case series of SARS-CoV-2 infection in the paediatric population and epidemiological data on COVID-19 published on official government websites internationally and in Australia.

\section{Results}

The CFR of SARS-CoV-2 pneumonia in children aged $<5$ years is estimated to be $0.15-1.35 \%$, which is lower than the estimated CFR of RSV pneumonia of $0.3-2.1 \%$, but higher than the estimated CFR of influenza pneumonia of $0.14-0.45 \%$.

\section{Discussion}

SARS-CoV-2 infection is likely to be less lethal than RSV in children aged $<5$ years, but more lethal than influenza.
AS AT 16 APRIL 2020, the COVID-19 pandemic, caused by the SARS-CoV-2 virus, is known to cause milder disease in children when compared with adults, based on a systematic review of epidemiological studies published to date. ${ }^{1}$ Nevertheless, children aged $<5$ years, and particularly those aged $<1$ year, are more vulnerable to severe disease. ${ }^{2}$

Overall, respiratory viral infections in young children have significant morbidity and mortality. In 2002, respiratory infections accounted for $18 \%$ of mortality for children aged $<5$ years. Morbidity is significant in this age group, accounting for $22 \%$ of hospitalisations and $59 \%$ of general practice consults in the UK. ${ }^{3}$

\section{Methods}

This review of the relevant literature and published epidemiological data from 18 March 2020 to 16 April 2020 aims to bring some clarity to the case fatality rates (CFRs) of SARS-CoV-2 infection and SARS-CoV-2 pneumonia in the paediatric population when compared with influenza, respiratory syncytial virus (RSV) and other serious vaccinepreventable pathogens.

This knowledge is especially relevant to general practitioners (GPs), as they are at the forefront of national childhood vaccination programs and play a critical part in promoting these programs.

\section{Definitions}

In this article, CFR is defined as the proportion of cases that are fatal within a specified time, in line with the definition from $A$ dictionary of epidemiology. ${ }^{4}$ Cases are defined as total cases (confirmed cases).

\section{Results}

\section{Estimating the CFR of SARS-CoV-2} infection in the paediatric population Data from China

In a case series of 2143 children aged $<18$ years, one death was recorded (an adolescent aged 14 years). ${ }^{2}$ As at 16 April 2020 , this is the largest case series of a paediatric population to date, based on nationwide data from the Chinese Center for Disease Control and Prevention.

In another case series, which had a sample size of 171 children aged $<16$ years, one death was recorded (an infant aged 10 months). ${ }^{5}$ In this particular study, 1391 children who were close contacts of confirmed and suspected cases were tested, with 171 positive cases. This study was based at Wuhan Children's Hospital, the only centre assigned by the central government for treating infected children aged $<16$ years in Wuhan.

\section{Data from Italy}

Based on a sample of 2341 children between the ages of zero and 18 years, one 
death of a child between the ages of zero and nine years was recorded. ${ }^{6}$ This equates to a crude CFR of $0.043 \%$. These data are based on information published online daily by the Istituto Superiore di Sanità.

\section{Data from the USA}

In a sample of 2572 children aged $<18$ years, three deaths were recorded. ${ }^{7}$ There was no information on the exact ages of these children. This was based on information published by the Centers for Disease Control and Prevention in their weekly Morbidity and Mortality Report.

Summary of the estimated CFR of SARSCoV-2 infection in the paediatric population

Based on the best available

epidemiological information available in the paediatric population (Table 1), the CFR of SARS-CoV-2 infection may range from $0.043 \%$ to $0.58 \%$ among a paediatric population aged $0-18$ years (Table 2).

The CFR of SARS-CoV-2 pneumonia may range from $0.10 \%$ to $0.90 \%$ in a paediatric population aged $<18$ years.

In the case series of 2143 children, the percentage of children aged $<5$ years with severe and critical disease was $8.71 \%$, while the percentage of children aged $<18$ years with severe and critical disease was $5.83 \% .^{2}$ Children aged $<5$ years are therefore 1.5 times more likely than children aged $<18$ years to have severe and critical disease.

Assuming that children who have severe and critical disease are more likely to die from COVID-19, it may be possible to derive a very crude estimate of the CFR of SARS-CoV-2 infection and SARS-CoV-2 pneumonia in children aged $<5$ years (Table 3).

\section{Estimating the CFR of RSV infection in the paediatric population}

$\mathrm{RSV}$ is the most common cause of death in childhood from pneumonia after Pneumococcus and Haemophilus influenzae type b. It accounts for $22 \%$ of all episodes of pneumonia in young children. ${ }^{8}$

A systematic review and meta-analysis of the global burden of disease in young children due to RSV estimated that globally in 2005:

- in developed countries, the CFR of RSV pneumonia of children aged $<1$ year was $0.7 \%$, while the CFR of children aged $<5$ years was $0.3 \%$

- in developing countries, the CFR of RSV pneumonia was $2.1 \%$ for both children aged $<1$ year and those aged $<5$ years.

Summary of the estimated CFR of RSV pneumonia in the paediatric population In the literature, the CFR of RSV is not available as most published articles focus only on RSV pneumonia or hospitalised patients with RSV.

Depending on age and geographical location, RSV pneumonia CFR may range from $0.3 \%$ to $2.1 \%$ in children aged $<5$ years (Table 2 ).

\section{Estimating the CFR of influenza infection in the paediatric population}

Influenza accounted for $7 \%$ of all severe paediatric pneumonia episodes in $2008 .^{9}$ It is the second most common pathogen in children with pneumonia, ${ }^{9}$ with the most common pathogen being RSV.
Based on information published by the Centers for Disease Control and Prevention (2017-18 influenza season $)^{10}$ and a systematic review and meta-analysis ${ }^{9}$ of influenza's global burden of disease of children (2008):

- the CFR of influenza infection may range from $0.0031 \%$ to $0.030 \%$ among a paediatric population aged $<5$ years

- the CFR of influenza-associated pneumonia may range from $0.14 \%$ to $0.45 \%$ in children aged $<5$ years (Table 2).

\section{Discussion}

In almost all epidemiological datasets available, a disproportionately low number of paediatric cases (aged $<18$ years) are reported. USA's Centers for Disease Control and Prevention reports $1.7 \% ;^{7}$ China reports $9 \% ;^{11}$ Italy reports $1.6 \% ;^{6}$ Korea reports $6.3 \%{ }^{12}$ and Australia reports $3.22 \% .^{13}$

Interestingly, a study on transmission dynamics in Shenzhen, China, found that children were as likely as adults to be infected, with rates of infection in children aged $<10$ years being $7.4 \%$, while the population average was $7.9 \% .^{14}$ The study looked at 391 positive cases and 1286 of their close contacts. ${ }^{14}$ Unfortunately, the study did not look specifically at the symptoms of the cases. Nevertheless, it is not uncommon for children with SARS-CoV-2 pneumonia to be asymptomatic. ${ }^{4,15}$

If it is true that the infection rate of children is similar to that of adults, the fact that they are disproportionately underrepresented in epidemiological studies may suggest that there are more

Table 1. Epidemiological data of the paediatric population from three different geographical locations

\begin{tabular}{|c|c|c|c|c|c|}
\hline Country & Data source & Sample size & Age range & CFR (SARS-CoV-2) & $\begin{array}{r}\text { CFR (SARS-CoV-2 } \\
\text { pneumonia) }\end{array}$ \\
\hline China $^{2}$ & Case series & 2,143 & $<18$ years & $0.047 \%$ & $0.10 \%$ \\
\hline China $^{5}$ & Case series & 171 & $<16$ years & $0.58 \%$ & $0.90 \%$ \\
\hline Italy 6 & Epidemiological data & 2,341 & $0-18$ years & $0.043 \%$ & Not available \\
\hline USA $^{7}$ & Epidemiological data & 2,572 & $<18$ years & $0.12 \%$ & Not available \\
\hline
\end{tabular}

CFR, case fatality rate 
cases of infected children who are undiagnosed than adults.

This may indicate that the true CFR of SARS-CoV-2 infection and SARS-CoV-2 pneumonia in children is much lower than what is suggested in this article.

Nevertheless, based on the estimates presented in Table 2, RSV pneumonia is more fatal than SARS-CoV-2 pneumonia and influenza-associated pneumonia.

There is no vaccine available for RSV, although it is likely to be more fatal than SARS-CoV- 2 in children aged $<5$ years. Palivizumab, a monoclonal antibody that has been shown to reduce hospitalisation rates, is available for high-risk children in Australia. ${ }^{16}$

While more epidemiological studies are required, this article will hopefully provide impetus for further research to more accurately quantify the lethality of SARS-CoV-2 pneumonia in young children, and spur efforts towards an RSV vaccine. Fortunately, there is already a widely available vaccine against invasive pneumococcal disease, which has a CFR of $5.6 \%$ in children aged $<5$ years, ${ }^{17}$ and H. influenzae type $b$, which has a CFR of $4.56 \%$ in a similar age group. ${ }^{18}$

\section{Limitations}

As a result of the limited availability of data and different testing criteria and preparedness of health systems in various countries, estimating the true CFR is challenging. As such, data from different countries are presented.

Data from government epidemiological sources are more likely to be underestimations of the CFR, as the numerator at the early phase of the pandemic is likely to be underestimated. Conversely, data from case series are more likely to be overestimations of the CFR, as the denominator is likely to be an underestimate of the true number of cases.

These are very crude estimates of the CFR of SARS-CoV-2 infection in children, based on the best available data.

Unfortunately, the US Centers for Disease Control and Prevention data and Italian data do not provide sufficient information on the number of infected children with COVID-19 pneumonia. As such, the CFR of SARS-CoV-2 pneumonia is only estimated using data from China.

Additionally, each case series or epidemiological dataset referenced had

Table 2. Comparison of estimated case fatality rate of SARS-CoV-2, respiratory syncytial virus and influenza in the paediatric population

\begin{tabular}{lcrr}
\hline & SARS-CoV-2 & $\begin{array}{c}\text { Respiratory } \\
\text { syncytial virus }\end{array}$ & Influenza \\
\hline Case fatality rate & $0.043-0.58 \%$ & Not available & $0.0031-0.030 \%$ \\
\hline $\begin{array}{l}\text { Case fatality rate } \\
\text { pneumonia) }\end{array}$ & $0.10-0.90 \%$ & $0.3-2.1 \%$ & $0.14-0.45 \%$ \\
\hline Age range & $0-18$ years & $<5$ years & $<5$ years \\
\hline
\end{tabular}

Table 3. Comparison of estimated case fatality rate of SARS-CoV-2, respiratory syncytial virus and influenza in children aged $<5$ years

\begin{tabular}{lccc} 
& SARS-CoV-2 & $\begin{array}{c}\text { Respiratory } \\
\text { syncytial virus }\end{array}$ & Influenza \\
\hline Case fatality rate & $0.065-0.87 \% *$ & Not available & $0.0031-0.030 \%$ \\
\hline $\begin{array}{l}\text { Case fatality rate } \\
\text { pneumonia) }\end{array}$ & $0.15-1.35 \% *$ & $0.3-2.1 \%$ & $0.14-0.45 \%$ \\
\hline Age range & $<5$ years & $<5$ years & $<5$ years \\
\hline *These figures have been obtained by multiplying those in Table 2 by 1.5. &
\end{tabular}

a different age cut-off. As such, it is not possible to provide accurate CFR estimates for children aged $<5$ years with SARS-CoV- 2 for a direct comparison with the systematic reviews and meta-analyses available for RSV and influenza in young children.

Data on comorbidities of the children were unavailable from all sources, hence it is not possible to ascertain CFRs based on pre-existing risk factors.

\section{Conclusion}

There are early indications that SARS-CoV-2 pneumonia in children aged $<5$ years may be more lethal than influenza, but not as lethal as RSV. Invasive pneumococcal disease and $H$. influenzae, both vaccine-preventable diseases, are at least twice as lethal as RSV.

This highlights the importance of early childhood vaccination programs and influenza vaccination in the midst of the COVID-19 pandemic. Parents should not avoid bringing their young children to general practice clinics, local council or pharmacies for vaccinations for fear of COVID-19, as many of these vaccinations protect their children from diseases more severe and deadly than COVID-19.

High-quality epidemiological studies, particularly in the paediatric population, will be vital to develop better estimates of the CFR of SARS-CoV-2 infection and pneumonia in young children.

\section{Author}

James Shunxian Wei MBBS, BMedSc, FRACGP, DCH, Principal GP, Standish Street Surgery, Vic; Visiting Medical Officer, Alpine Health, Vic. james.wei.standish@gmail.com

Competing interests: None.

Funding: None.

Provenance and peer review: Not commissioned, externally peer reviewed.

\section{References}

1. Ludvigsson JF. Systematic review of COVID-19 in children shows milder cases and a better prognosis than adults. Acta Paediatr 2020;109(6):1088-95. doi: 10.1111/apa.15270.

2. Dong $Y$, Mo X, Hu Y, et al. Epidemiology of COVID-19 among children in China. Pediatrics 2020;145(6):e20200702. doi: 10.1542/peds.20200702

3. Tregoning JS, Schwarze J. Respiratory viral infections in infants: Causes, clinical symptoms, virology, and immunology. Clin Microbiol Rev 2010:23(1):74-98. doi: 10.1128/CMR.00032-09. 
4. Porta M. A dictionary of epidemiology. 5th edn. New York, NY: Oxford University Press, 2008.

5. Lu X, Zhang L, Du H, et al. SARS-CoV-2 infection in children. N Engl J Med 2020;382(17):1663-65. doi: 10.1056/NEJMc2005073.

6. EpiCentro. Integrated surveillance of COVID-19 in Italy. Rome, IT: EpiCentro, 2020.

7. Bialek S, Gierke R, Hughes M, McNamara LA, Pilishvili T, Skoff T. Coronavirus disease 2019 in children - United States, February 12-April 2, 2020. MMWR Morb Mortal Wkly Rep 2020;69(14):422-26. doi: 10.15585/mmwr.mm6914e4.

8. Nair H, Nokes DJ, Gessner BD, et al. Global burden of acute lower respiratory infections due to respiratory syncytial virus in young children: A systematic review and meta-analysis. Lancet 2010;375(9725):1545-55. doi: 10.1016/S01406736(10)60206-1.

9. Nair H, Brooks WA, Katz M, et al. Global burden of respiratory infections due to seasonal influenza in young children: A systematic review and meta-analysis. Lancet 2011;378(9807):1917-30. doi: 10.1016/S0140-6736(11)61051-9.

10. Centers for Disease Control and Prevention. 2017-2018 estimated influenza illnesses, medical visits, and hospitalizations, and deaths and estimated influenza illnesses, medical visits, hospitalizations, and deaths averted by vaccination in the United States. Atlanta, GA: CDC, 2019.
11. Wu Z, McGoogan JM. Characteristics of and important lessons from the coronavirus disease 2019 (COVID-19) outbreak in China: Summary of a report of 72314 cases from the Chinese center for disease control and prevention. JAMA 2020;323(13):1239-42. doi: 10.1001/ jama.2020.2648.

12. Korean Centre for Disease and Control and Prevention. Press release. Cheongju-si, Chungbuk: KCDC, 2019. Available at www.cdc.go.kr/board/ board.es? $\mathrm{mid}=$ a30402000000 \&bid $=0030$ [Accessed 24 July 2020].

13. COVID-19 National Incident Room Surveillance Team. COVID-19, Australia: Epidemiology report 10 (reporting week to 23:59 AEST 5 April 2020). Commun Dis Intell (2018) 2020;44. doi: 10.33321/ cdi.2020.44.30

14. Bi Q, Wu Y, Mei S, et al. Epidemiology and transmission of COVID-19 in 391 cases and 1286 of their close contacts in Shenzhen, China: A retrospective cohort study. Lancet Infect Dis 2020;20(8):911-19. doi: 10.1016/S14733099(20)30287-5.

15. Chan JFW, Yuan S, Kok KH, et al. A familial cluster of pneumonia associated with the 2019 novel coronavirus indicating person-to-person transmission: A study of a family cluster. Lancet 2020;395(10223):514-23. doi: 10.1016/S01406736(20)30154-9.
16. Wang D, Cummins C, Bayliss S, Sandercock J, Burls A. Immunoprophylaxis against respiratory syncytial virus (RSV) with palivizumab in children: A systematic review and economic evaluation. Health Technol Assess 2008;12(36):iii,ix-x,1-86. doi: 10.3310/hta12360.

17. Backhaus E, Berg S, Andersson R, et al. Epidemiology of invasive pneumococcal infections: Manifestations, incidence and case fatality rate correlated to age, gender and risk factors. BMC Infect Dis 2016;16:367. doi: 10.1186/s12879-0161648-2.

18. Watt JP, Wolfson LJ, O'Brien KL, et al. Burden of disease caused by Haemophilus influenzae type b in children younger than 5 years: Global estimates. Lancet 2009:374(9693):903-11. doi: 10.1016/ S0140-6736(09)61203-4.

correspondence ajgp@racgp.org.au 